medRxiv preprint doi: https://doi.org/10.1101/2020.06.26.20123216; this version posted November 9, 2020. The copyright holder for this preprint (which was not certified by peer review) is the author/funder, who has granted medRxiv a license to display the preprint in

All rights reserved. No reuse allowed without permission.

\title{
Deep ensemble multitask classification of emergency medical call incidents combining multimodal data improves emergency medical dispatch
}

Pablo Ferri ${ }^{1}$, Carlos Sáez ${ }^{1}$, Antonio Félix-De Castro ${ }^{2}$, Javier Juan-Albarracín ${ }^{1}$, Vicent Blanes-Selva ${ }^{1}$, Purificación Sánchez-Cuesta ${ }^{2}$ and Juan M García-Gómez ${ }^{1}$

${ }^{1}$ Biomedical Data Science Laboratory (BDSLab), Instituto de Aplicaciones de las Tecnologías de la Información y de las Comunicaciones Avanzadas (ITACA), Universitat Politècnica de València (UPV), Valencia, Spain

${ }^{2}$ Conselleria de Sanitat Universal i Salut Pública, Generalitat Valenciana (GVA), Valencia, Spain

Corresponding Author: pabferb2@upv.es

\begin{abstract}
The objective of this work was to develop a predictive model to aid non-clinical dispatchers to classify emergency medical call incidents by their life-threatening level (yes/no), admissible response delay (undelayable, minutes, hours, days) and emergency system jurisdiction (emergency system/primary care) in real time. We used a total of 1244624 independent incidents from the Valencian emergency medical dispatch service in Spain, compiled in retrospective from 2009 to 2012, including clinical features, demographics, circumstantial factors and free text dispatcher observations. Based on them, we designed and developed DeepEMC ${ }^{2}$, a deep ensemble multitask model integrating four subnetworks: three specialized to context, clinical and text data, respectively, and another to ensemble the former. The four subnetworks are composed in turn by multi-layer perceptron modules, bidirectional long short-term memory units and a bidirectional encoding representations from transformers module. DeepEMC ${ }^{2}$ showed a macro F1score of 0.759 in life-threatening classification, 0.592 in admissible response delay and 0.757 in emergency system jurisdiction. These results show a substantial performance increase of $12.5 \%, 17.5 \%$ and $5.1 \%$, respectively, with respect to the current in-house triage protocol of the Valencian emergency medical dispatch service. Besides, DeepEMC ${ }^{2}$ significantly outperformed a set of baseline machine learning models, including naive bayes, logistic regression, random forest and gradient boosting ( $\alpha=0.05)$. Hence, DeepEMC ${ }^{2}$ is able to: 1) capture information present in emergency medical calls not considered by the existing triage protocol, and 2) model complex data dependencies not feasible by the tested baseline models. Likewise, our results suggest that most of this unconsidered information is present in the free text dispatcher observations. To our knowledge, this study describes the first deep learning model undertaking emergency medical call incidents classification. Its adoption in medical dispatch centers would potentially improve emergency dispatch processes, resulting in a positive impact in patient wellbeing and health services sustainability.
\end{abstract}

Keywords: medical emergencies, emergency medical calls, emergency medical dispatch, deep learning, ensemble learning, multitask learning. 
medRxiv preprint doi: https://doi.org/10.1101/2020.06.26.20123216; this version posted November 9, 2020. The copyright holder for this preprint (which was not certified by peer review) is the author/funder, who has granted medRxiv a license to display the preprint in perpetuity.

All rights reserved. No reuse allowed without permission.

\section{Introduction}

Emergency medical dispatch (EMD) involves the reception and management of requests for medical assistance in an emergency medical services system [1]. It comprises two main dimensions: call-taking, where emergency medical calls are received and incidents are classified according to their priority-triaged - and controlling, where the best available resources are dispatched to handle the event [2].

The call-taking process is generally managed by emergency medical dispatchers [3]. These mediators are in many cases non-clinical staff, trained with the essential knowledge of medical emergencies for the proper and efficient management of the incident $[1,4]$. Dispatchers usually follow a clinical protocol, established in the medical dispatch center, and periodically verified by medical supervisors [5].

However, despite preparation and the existence of triage protocols, assigning priorities to emergency medical call incidents (EMCI) is a challenging and stressful task for dispatchers, requiring constant concentration [6-8]. Additionally, there is always an inherent uncertainty on the real patient state, since the information of the event is gathered from telephonic interview processes. Furthermore, there are time constraints due to the incident priority or the need for tackling other incoming calls [9]. A wrong priority assignment derives either in insufficient medical attention or unnecessary resource deployment [10-12]. In consequence, EMCIs triage protocols are continuously revised and enhanced.

Many triage algorithms, such as the Emergency severity index [13], the Manchester triage system [14], the Canadian triage and acuity scale [15] or the Australasian triage scale [16], have been widely studied and enriched [17-20]. However, they are difficult to benchmark, deriving in no international agreement about their use for EMD [21]. Likewise, these algorithms depend on structured clinical information which is not always available during the call [22]. As such, improvements in EMD processes by redefining this sort of protocols are extremely costly and limited.

In the Valencian Community (Spain), the triage of EMCI is currently supported by an in-house triage protocol, based on a clinical decision tree, grounded on heavily structured clinical variables, e.g., chest pain (yes or no), collected throughout the interview in a sequential manner. Therefore, free text dispatcher observations, with higher expressiveness than structured data, cannot be automatically processed by the protocol, limiting its generalization to situations beyond the established guidelines.

The potential capability of deep learning to enhance EMCI classification through the provision of decision support to non-clinical dispatchers, was spotted by the Health Services Department of the Valencian region, aware of the potential of these models: deep learning is at the state of the art of machine learning in tasks involving complex types of data [23], e.g., high dimensional, unstructured, sequential, multimodal [2427], such as those found in EMCI databases. Likewise, this and other machine learning tools have already been applied to tackle EMD challenges such as ambulance allocation [28-30], prediction of emergency calls volume [31], automatic stress detection of the caller [32], interpretable knowledge extraction [33], performance monitoring [34], cardiac arrest calls assistance [35] or triaging unconscious and fainting patients [36]. Therefore, we can argue that deep learning models are a feasible and promising technology to improve EMD through EMCI classification.

In this work, we develop and evaluate a deep learning model to provide decision support to non-clinical dispatchers in EMCI triage from the medical dispatch center of the Valencian region. Our model is designed to integrate the EMCI data collected during the call and carry out its classification. Despite of the existence of studies dealing with EMCI classification for specific disorders, as mentioned in the previous paragraph, to our knowledge, this is the first largescale study undertaking a general EMCI classification trough deep learning.

\section{Materials}

\subsection{Dataset}

\subsubsection{Overview}

A total of 1244624 independent EMCI of the Health Services Department of the Valencian Community, were compiled in retrospective from 2009 to 2012. The Health Services Department board of the Valencian Community approved the data use for this project, removing before their analysis any information that may disclose the identity of the person.

These EMCI data included during-call and after-call data. We categorized the data variables as structured-fixed fields-and unstructured-open fields-as well as stationary—with no implicit order — and sequential—with an implicit order (Figure 1). 
medRxiv preprint doi: https://doi.org/10.1101/2020.06.26.20123216; this version posted November 9, 2020. The copyright holder for this preprint (which was not certified by peer review) is the author/funder, who has granted medRxiv a license to display the preprint in perpetuity.

All rights reserved. No reuse allowed without permission.

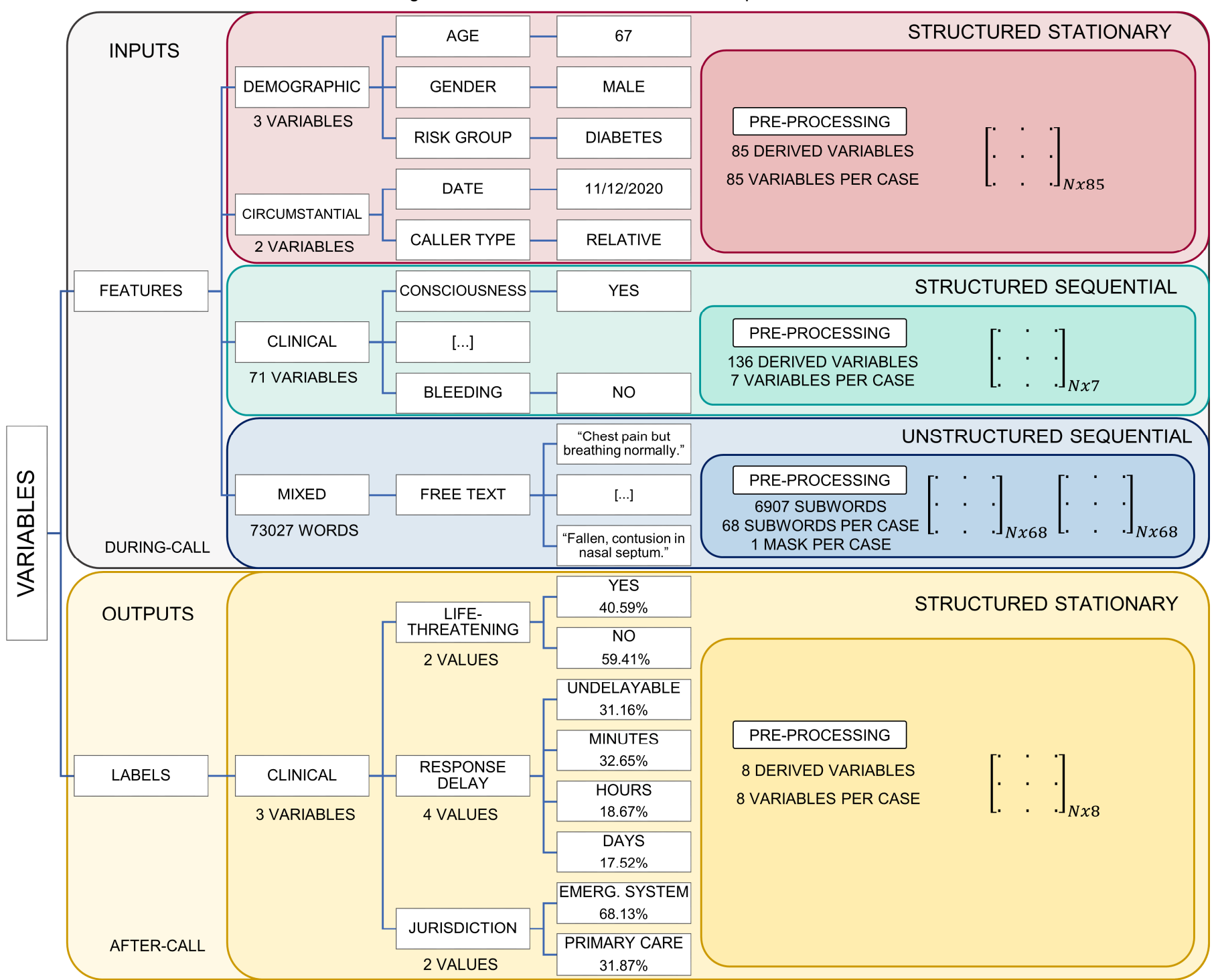

Figure 1. Dataset variables arranged by type. Names and cardinality, before and after pre-processing (derived variables), are presented, indicating how many variables_ or subwords, when referring to text features-are available per case after pre-processing. Examples for their values are also included. Class frequencies for each output label are also reported. N is equal to the 722270 EMCI used in the study.

\subsubsection{During-call data}

During-call data (Figure 1 top) are recorded during the emergency medical call. These data consist of demographics, circumstantial factors, clinical featurescollected throughout the triage tree navigation-and free text dispatcher observations:

Demographics data-structured and stationaryinclude age, gender and risk group variables. Age is a numerical discrete feature, gender is a categorical binary variable (male, female) and risk group is a categorical multiclass variable-with multiple possible values, such as asthmatic, allergic, cardiac, diabetic, neoplastic, etc.

Circumstantial factors data-structured and stationary-include date and caller type variables. The latter consists on a categorical multiclass variable, keeping information about the person or institution which made the emergency medical call and taking values such as police, red cross, the patient, a relative, etc.
Clinical variables data-structured and sequentialinclude features providing relevant medical information. They are collected in a sequential manner during the call, registering a subset of them, from the total 71 variables available. A full list including all these variables is available in Table 1. These variables are categorical, presenting one possible value or multiple ones. An example of how four clinical variables and their values are registered during an emergency medical call could be: previous trauma, yes; hemorrhage, yes; bleeding site, rectal bleeding; consequences of the clinic, severe blood loss.

Finally, free text dispatcher observationsunstructured and sequential-consist on short sentences, written during the call and providing additional relevant information which cannot be recorded in a structured manner. The language in which they are written is Spanish. Examples of two free text dispatcher observations bound each one to a different event are (translated into English): according to the caller epileptic crisis, he has drunk and taken pills, he is half- 
medRxiv preprint doi: https://doi.org/10.1101/2020.06.26.20123216; this version posted November 9, 2020. The copyright holder for this preprint (which was not certified by peer review) is the author/funder, who has granted medRxiv a license to display the preprint in perpetuity.

All rights reserved. No reuse allowed without permission.

Table 1. Clinical variables with some of their example values. Certain variables have just one possible associated value, while others may exhibit multiple values. To ease presentation, example values are limited to three in this table.

\begin{tabular}{|c|c|c|c|}
\hline Variable & Example values & Variable & Example values \\
\hline Active arrhythmia & yes & ICTUS code criteria & no \\
\hline Active suicide attempt & yes & Impaired consciousness & yes \\
\hline \multicolumn{4}{|l|}{ Acute decompensation of mental } \\
\hline illness & yes & Impaired consciousness level & yes \\
\hline & & & highway, inter-urban road, lakes or rivers \\
\hline Administration of medication & yes & Incident location & and other inland waters \\
\hline Age & less than 1 year, over 70 years & Injury severity & major, minor, moderate \\
\hline \multirow[t]{2}{*}{ Altered behavior } & abnormal behavior, aggressiveness/agitation & Intake household product & yes \\
\hline & & Intake of substance (medicine or & \\
\hline Arterial vascular clinic & yes & toxic) & yes \\
\hline Bleeding site & epistaxis, hematuria, melena & Itchiness & yes \\
\hline Blood glucose & abnormal & Medical history & cardiac pathology, copd, diabetes \\
\hline Blood or mucus in stool & no, yes & Menstruation & yes \\
\hline Breathing & absent, labored & Nasal congestion & no, yes \\
\hline Burn & yes & Number of injured & from 1 to 3 , over 3 \\
\hline Causation of intake & autolysis attempt, medication error & Ongoing birth & yes \\
\hline Choking & yes & Pain & abdomen, generalized, head, lumbar area \\
\hline Clinic start & abrupt, progressive & Pregnant & no, yes \\
\hline Clinic triggers & upsetting & Previous trauma & no, yes \\
\hline \multirow[t]{2}{*}{ Clinical evolution } & stable without worsening & Prior care & no, yes \\
\hline & mild blood loss, moderate blood loss, severe & & \\
\hline Consequences of the clinic & blood loss & Recovered unconscious & yes \\
\hline & & & impossible to obtain, insulin, oral \\
\hline Constipation & yes & Regular medication & antidiabetics \\
\hline Consumption of toxic substances & yes & Relationship and contact level & absent, present \\
\hline Cyanosis & yes & Seizures & yes \\
\hline Death & yes & Sickness & yes \\
\hline Diarrhea & yes & Signs of severity & no, yes \\
\hline Dizziness & yes & Skin alteration type & edema/swelling \\
\hline Drug intake & no, yes & Skin disorders & yes \\
\hline Dyspnoea & no, yes & Symptoms of glottic edema & yes \\
\hline Dysuria and / or hematuria & yes & Time of evolution & over 24 hours \\
\hline Eating / bilious vomiting & yes & Toxic substance & heroin \\
\hline Epidemiological criteria & $\begin{array}{c}\text { contact with contaminated samples, contact } \\
\text { with diagnosed cases }\end{array}$ & Treatment & $\begin{array}{l}\text { prescribed treatment for the clinical picture, } \\
\text { psychiatric medication }\end{array}$ \\
\hline \multicolumn{4}{|l|}{ Epidemiological infectious } \\
\hline disease & yes & Type of accident & aggression, collision, drowning \\
\hline Existence of neurological focality & yes & Unconscious & no, yes \\
\hline Fever & over 38 , over 39 & Vegetative picture & no, yes \\
\hline Flu syndrome & yes & Venous vascular clinic & yes \\
\hline Gastrointestinal symptoms & yes & Vomiting & yes \\
\hline Hemorrhage & no, yes & Without further information & yes \\
\hline Hypertensive crisis & yes & & \\
\hline
\end{tabular}

conscious with half-closed eyes; patient bleeds abundantly from the head after falling at home, they have just found it in a pool of blood.

\subsubsection{After-call data}

After-call data are recorded at a time after the call and used to derive EMCI classification labels, since they provide reliable up-to-date information about the real patient state. These data include: posterior physician diagnosis, standardized by International classification of diseases codes [37], such as syncope (ICD 780.2) or acute myocardial infarction (ICD 410); maneuvers and procedures indicating if the patient was intubated, reanimated, sedated, received surgery, etc.; and hospitalizations and urgency stays with information about the department where the patient was treated, the amount of time he stayed there and his discharge code.

\subsubsection{Labels derivation}

We transcribed the information contained in aftercall data to three different and complementary EMCI classification labels (Figure 1 bottom): life-threatening level (yes/no), admissible response delay (undelayable, minutes, hours, days) and emergency system jurisdiction (emergency system/primary care). The mapping between after-call data and EMCI classification labels was established by a panel of 17 physicians from the Health Services Department of the Valencian Community, using a Delphi methodology [38]. 
medRxiv preprint doi: https://doi.org/10.1101/2020.06.26.20123216; this version posted November 9, 2020. The copyright holder for this preprint (which was not certified by peer review) is the author/funder, who has granted medRxiv a license to display the preprint in perpetuity.

All rights reserved. No reuse allowed without permission.

\subsubsection{Data quality assessment and inclusion criteria}

To ensure the highest reliability of the model training data, we performed and reported a data quality analysis on the included data [39]. The analysis included the assessment of data quality dimensions of completeness and consistency, as well as temporal and multi-source variability [40-42]changes in the statistical distributions of data over time or among sources, respectively. The main findings included: approximately $30 \%$ of data with at least one missing label; and outlying distributions in some dispatchers, especially those with less than 100 calls.

According to these results, we considered, for the next stages of our work, those EMCI which after-call data were fully available, and which during-call data were registered by non-novice dispatchers - dispatchers with more than 100 calls managed. The final working dataset size comprised 722270 EMCI.

\subsection{Framework}

The implementation language was Python 3.7.3 [43], making use of libraries Pandas [44], NumPy [45], and Fuzzywuzzy [46], for data pre-processing and Sklearn [47], Pytorch (version 1.4.0) [48], Hugginface transformers [49] and Hyperopt [50] for modeling.

\section{Methods}

\subsection{Data pre-processing}

Depending on variable type, different pre-processing techniques were applied, mapping the original data to a matrix representation to be used for the deep learning model (Figure 1 right, highlighted pre-processing blocks):

Age, a structured stationary discrete ordinal variable, was mapped to a fuzzy [51] representation trough piecewise linear functions [52]. These membership functions, represented in Figure 2, were validated by physicians of the Health Services Department of the Valencian Community. This smoothing transformation was carried out to avoid sharp transitions derived from grouping in a small set of categories discrete ordinal variables with high cardinality in their values.

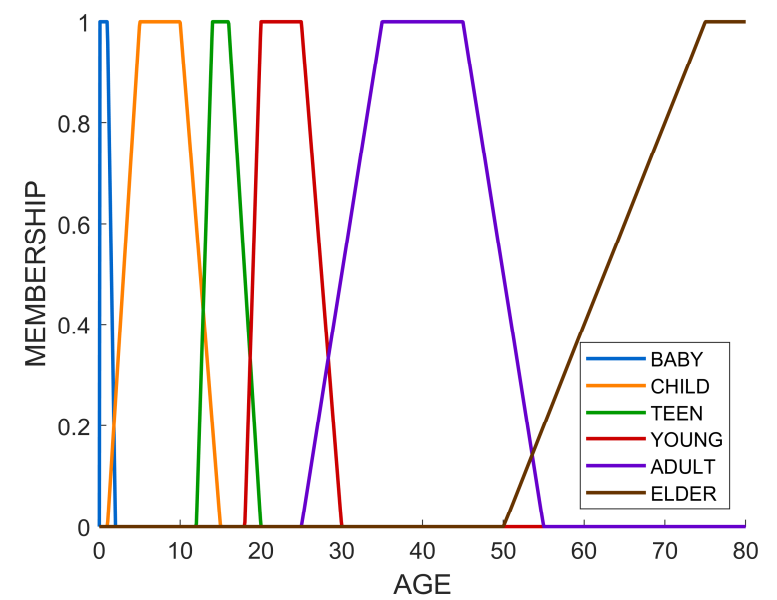

Figure 2. Piecewise linear functions representing age group membership.
Gender, risk group and caller type, structured stationary categorical variables, were one-hot encoded while several variables were derived from the date variable: weekday, month, if the day was or not a weekend day and if the day was or not was a bank holiday. These resulting variables, also structured stationary categorical variables, were one-hot encoded too.

Regarding the clinical variables, structured sequential variables, each variable-value pair was converted to an integer, conforming then, sequences of integers that were pre-padded afterwards, to ensure sequences of fixed length [53]. This length was equal to 7 , since in more than $99 \%$ of the incidents reported, the number of clinical variables collected was equal or lower than 7 .

Spelling correction processes by means of fuzzy string matching [54] were applied to the free text dispatcher observations, unstructured sequential variables, to reduce vocabulary dimensionality and noise. Besides, subword tokenization with WordPiece was carried out to reduce vocabulary size [55]. To ensure sequences of fixed length while keeping information about the original sequences lengths, post-padding and attention mask generation were conducted. The padding length was set in 68 , since in more than $99 \%$ of the incidents reported, the number of subwords written was equal or lower than 68 .

Finally, labels, structured stationary categorical data, were one-hot encoded, deriving in a label matrix of 8 columns, each one associated with a specific label-class pair.

\subsection{Data splitting and sampling}

To evaluate model performance and tune hyperparameters without any bias, data were iteratively and randomly split into six subsets (Figure 3) [56]. First, data were randomly split into two disjoint design and test sets, with $80 \%$ and $20 \%$ proportions respectively. Next, the design set was randomly divided again into a training and a validation set, with $80 \%$ and $20 \%$ proportions. Finally, a sampling step was performed taking 100000 elements to define a training and a validation sample.

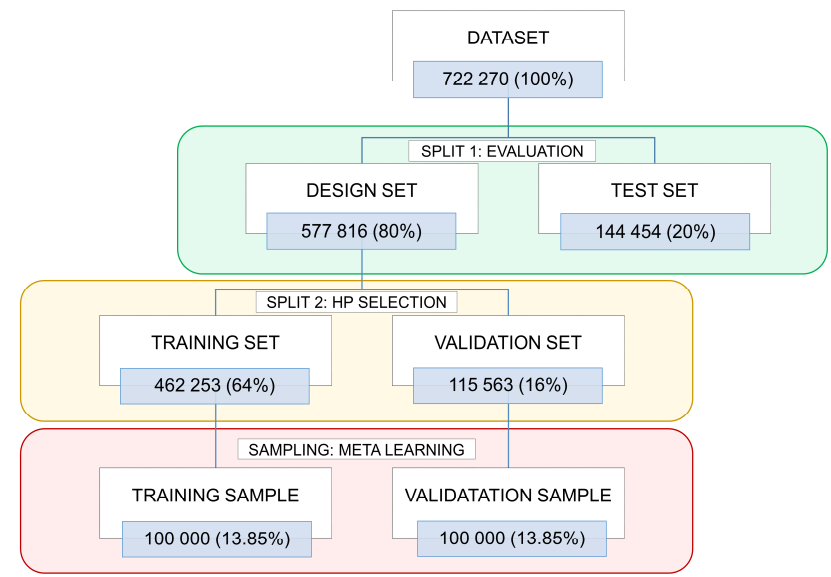

Figure 3. Data splitting and sampling. The number of data of each partition, along with its percentage respect the total number of data, are provided. Abbreviations: HP, hyperparameter. 
medRxiv preprint doi: https://doi.org/10.1101/2020.06.26.20123216; this version posted November 9, 2020. The copyright holder for this preprint (which was not certified by peer review) is the author/funder, who has granted medRxiv a license to display the preprint in perpetuity.

\section{All rights reserved. No reuse allowed without permission.}

\subsection{Deep neural network design}

The problem of classifying EMCI combining multimodal data was divided into four subproblems: three EMCI classification problems taking as inputs for each one EMCI data from the same type-structured stationary, structured sequential and unstructured sequential —and a last EMCI classification problem taking as inputs inner outputs obtained from the solution of the prior problems. To solve these four challenges, four deep learning (DL) subnetworks were developed: the Context subnetwork (ConNet), the Clinical subnetwork (CliNet), the Text subnetwork (TextNet) and the Ensemble subnetwork (EnsNet). Finally, once trained, they were combined in a single global modular neural network model [57].

Likewise, as the life-threatening, response delay and jurisdiction labels provide different but related information, e.g., a life-threatening situation implies a low admissible response delay, a multitask learning [58] paradigm was followed, to exploit these label dependences. To promote training efficiency and regularization while reducing the number of subnetworks parameters, a hard parameter sharing approach [59] was adopted. Hence, each of the four developed subnetworks presented a task-shared block-same set of parameters for all label prediction tasks-and a task-specific block-specific set of parameters for each label prediction task.

The ensemble of the four multitask subnetworks defined DeepEMC ${ }^{2}$ - Deep Ensemble Multitask Classifier for Emergency Medical Calls-the global and definitive DL model.

Next, we describe in detail each of the subnetworks integrated in DeepEMC ${ }^{2}$, supported by Figure 4:

The Context subnetwork (Figure 4 left) deals with the demographics and circumstantial factors bound to an EMCI. It consists on a multi-layer perceptron (MLP) [60] due to its adequateness to model structured and stationary data, composed by dense and output blocks. A dense block integrates a fully connected layer [61] a batch normalization layer [62] to manage internal covariate shift, a leaky ReLU [63] activation function to avoid vanishing and exploding gradients, while preventing dead neurons issues [64] and a dropout layer [65] to prevent neuron co-adaptation. An output block is composed by a fully connected layer and a softmax activation function, to dispose of a normalization scorebetween 0 and 1 - for each class of each predicted label.

The Clinical subnetwork (Figure 4 center) deals with the clinical features collected during the call. It consists on a recurrent model, since clinical features are notified in a sequential manner, being their recording order potentially informative. It is composed by an embedding layer [66], which compresses the sparse input space into a smaller and dense one; a stack of multiple bidirectional long short-term memory (BLSTM) [67] units, which capture long-term dependences far better than standard recurrent models; multiple skip connections [68] across the BLSTM units, to reduce the risk of losing relevant information during BLSTM propagation; a concatenation block-concatenates the outputs of these skip connections-and a MLP module, integrated by dense and output blocks, to act as an intermediary between the multiple BLSTM outputs and the final label predictions.

The Text subnetwork (Figure 4 right) deals with the free text dispatcher observations-unstructured and sequential-written during an EMCI. It is composed by a bidirectional encoding representations from transformers (BERT) [69] block, since this model is at the state of the art in natural language processing tasks, including text classification, and a MLP module, to relate BERT outputs with label outputs. The BERT clock is comprised in turn by an embedding block, an encoder block [70], and a pooler block, while the MLP component is constituted by dense and output blocks.

The Ensemble subnetwork (Figure 4 bottom) integrates inner outputs from the ConNet, the CliNet and the TextNet to generate the final outputs of DeepEMC ${ }^{2}$. It consists of a concatenation block with a MLP component, composed by dense and output blocks. The inputs of the concatenation block are the outputs of the last layer of the dense block prior to the task-specific block of each one of the former subnetworks. It takes these inner outputs, and not the final output scores since these last values aggregate tons of information in just a small set of scalar values; hence, the modeling potential of the inner outputs is higher.

\subsection{Parameter tuning}

Subnetworks were trained in a constructive modularized manner [57], so they were independently trained and assembled later as loosely coupled models. The optimizer selected for that was ADAM [71], given its learning adaptability, noisy gradients management and learning process stability [72,73]. A term of weight decay [74] was included in the parameters upgrading rule expression, to promote regularization. Likewise, it was followed a minibatch upgrading approach [75], computing gradients with backpropagation [76] and backpropagation through time [77]. The objective function was a cross-entropy [78] loss (CEL). For each subnetwork, three CEL were calculated-one per label-averaged afterwards and finally backpropagated to carry out the parameter tuning process. Layers with leaky ReLU activation functions were initialized with Kaiming initialization [79], while softmax activation function layers were initialized with Xavier's initialization [80]. 
medRxiv preprint doi: https://doi.org/10.1101/2020.06.26.20123216; this version posted November 9, 2020. The copyright holder for this preprint (which was not certified by peer review) is the author/funder, who has granted medRxiv a license to display the preprint in perpetuity.

All rights reserved. No reuse allowed without permission.

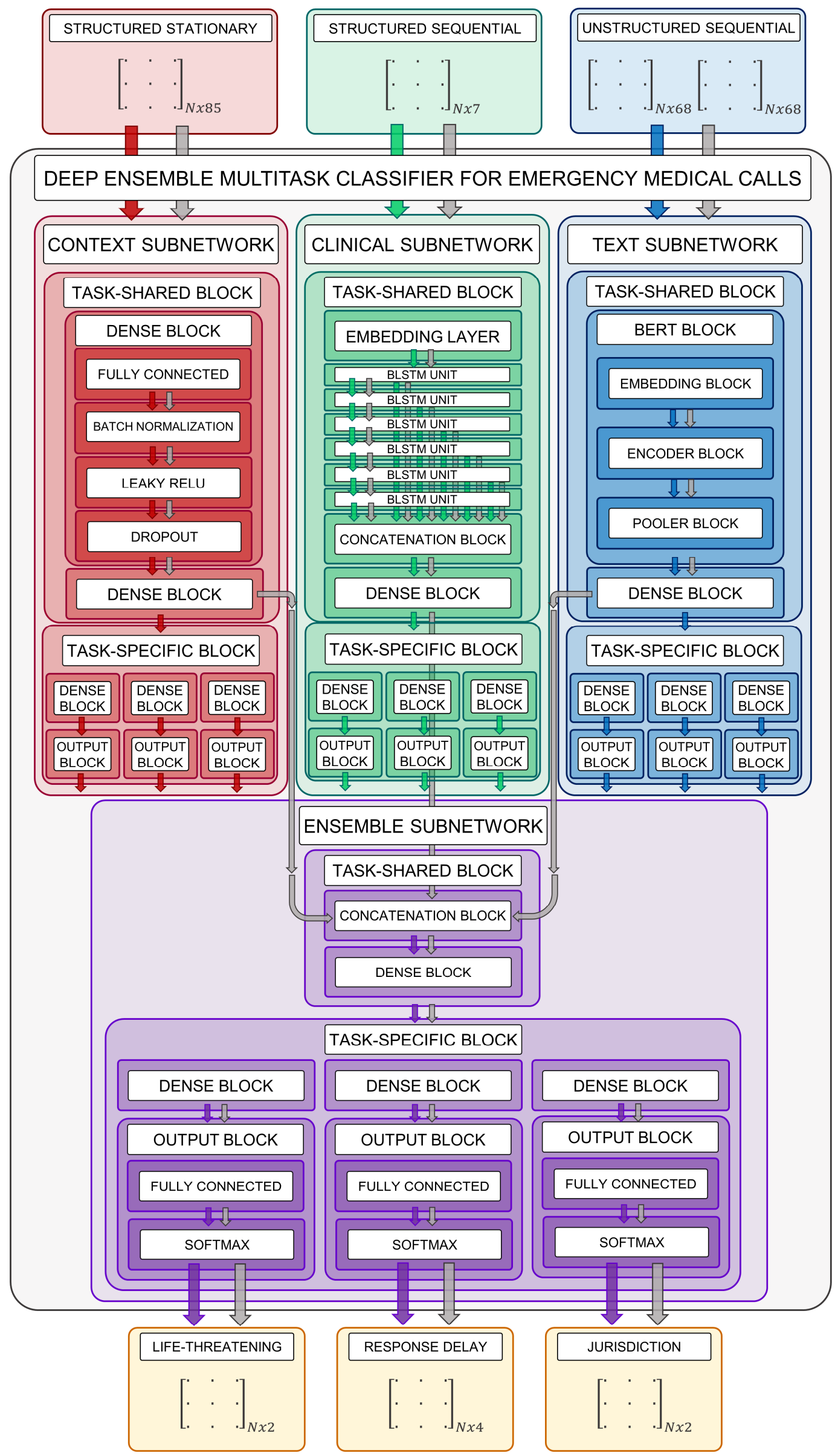

Figure 4. DeepEMC ${ }^{2}$ - Deep Ensemble Multitask Classifier for Emergency Medical Calls—architecture, including its constituting subnetworks-the Context subnetwork, the Clinical subnetwork, the Text subnetwork and the Ensemble subnetwork. Arrows indicate the forward propagation direction, for each subnetwork, as well as the global network (DeepEMC ${ }^{2}$ ), colored according to the particular neural network they refer. 
medRxiv preprint doi: https://doi.org/10.1101/2020.06.26.20123216; this version posted November 9, 2020. The copyright holder for this preprint (which was not certified by peer review) is the author/funder, who has granted medRxiv a license to display the preprint in perpetuity.

All rights reserved. No reuse allowed without permission.

\subsection{Hyperparameter tuning}

The influence of hyperparameters over subnetworks performance was carefully considered in this work, in order to maximize the attainable outcomes. The hyperparameters studied were related with subnetworks architecture and optimizer settings. These hyperparameters, as well as its definitive (optimal) values are presented in Table 2.

Hyperparameters were tuned following a multi-step strategy (Figure 5):

The first step involved an automatic active learning [81] hyperparameter optimization process (Figure 5 top): four surrogate models-one per subnetwork-based on treestructured parzen estimators [82], learned the conditional probability distribution of subnetworks hyperparameters given their associated CEL. Aiming to maximize the Expected Improvement [83] of the CEL, new hyperparamter configurations were iteratively sampled from the surrogate models, being upgraded after each training loop. Thereby, 280 different subnetworks-70 hyperparameter configurations times four subnetworks-were trained and evaluated in the training and validation samples, respectively.

Next, the best hyperparameter configurations proposed by the surrogate models were selected (Figure 5 middle). To prevent overfitting, the best five hyperparameter configurations for each subnetwork were taken to retrain and validate the subnetworks, in the training and the validation set, respectively, obtaining a total of 20 models trained in this step. Then, the CEL was obtained for each of them and those hyperparameter configurations with the best value-lowest validation CEL-were considered as the optimal hyperparameter configuration.

Finally, the optimal hyperparameters were used to retrain the four subnetworks using the whole design set, to ensure a proper exploitation of the data (Figure 5 bottom). Once trained, its integration into a single architecture defined DeepEMC ${ }^{2}$ - the global network - evaluated later in the test set.

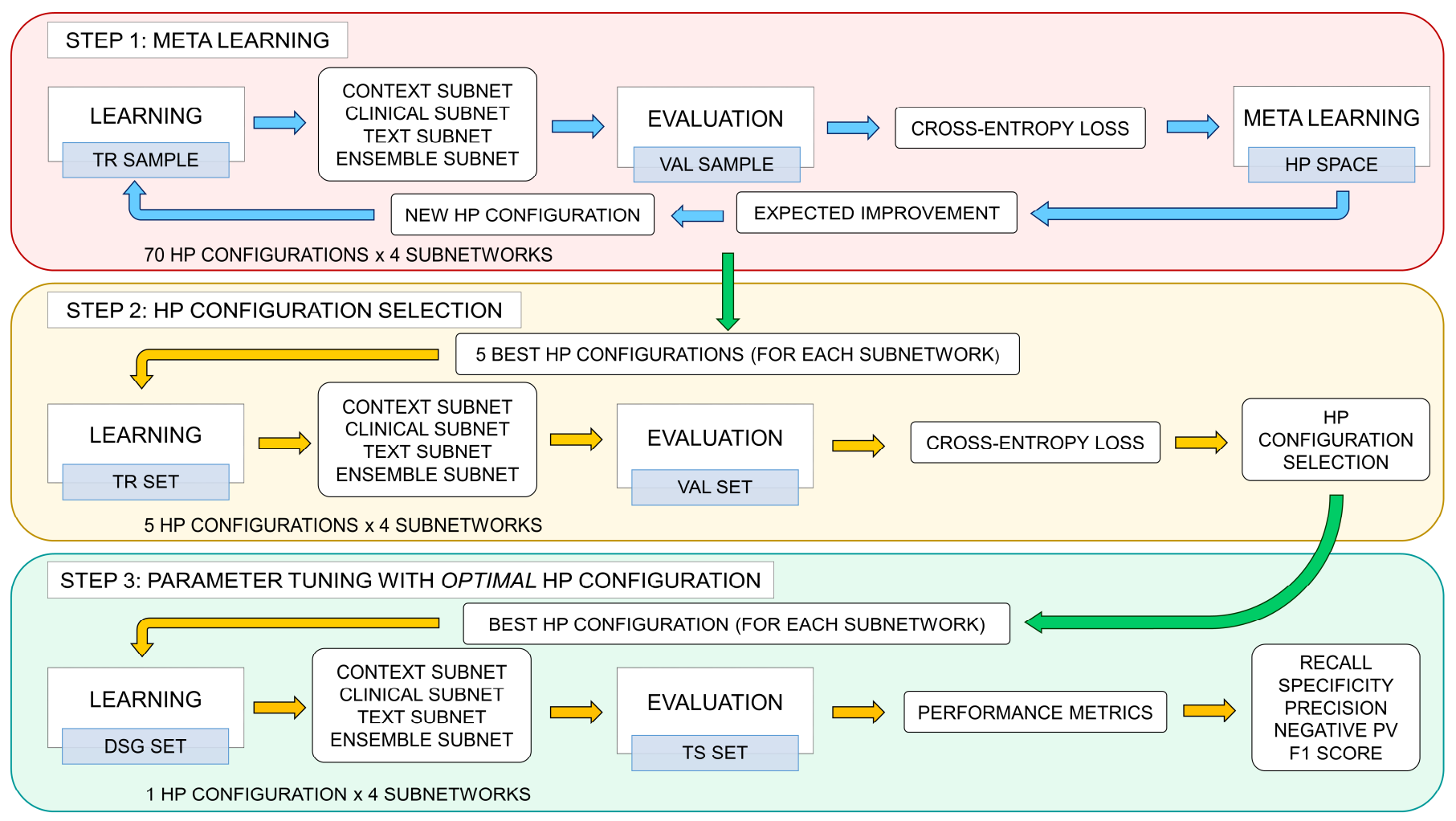

Figure 5. Multi-step hyperparameter tuning strategy. Yellow arrows imply unidirectionality, while blue arrows stand for a feedback loop, both inside a hyperparameter optimization step. Green arrows denote unidirectionality across hyperparameter optimization steps. Abbreviations: HP, hyperparameter; TR, training; VAL, validation; DSG, design TS, test. 
medRxiv preprint doi: https://doi.org/10.1101/2020.06.26.20123216; this version posted November 9, 2020. The copyright holder for this

preprint (which was not certified by peer review) is the author/funder, who has granted medRxiv a license to display the preprint in perpetuity.

All rights reserved. No reuse allowed without permission.

Table 2. Subnetworks hyperparameters with their definitive values obtained after carrying out the multi-step hyperparameter tuning process.

\begin{tabular}{ccccc}
\hline & \multicolumn{4}{c}{ Deep learning model subnetworks } \\
\cline { 2 - 5 } Hyperparameters & Context subnet & Clinical subnet & Text subnet & Ensemble subnet \\
\hline Embedding dimension & - & 8 & 96 & - \\
DB hidden layers & 1 & 1 & - & 1 \\
DB neurons per layer & 64 & 64 & - & 256 \\
DB dropout & 0.1 & 0.1 & - & 0.25 \\
BLSTM layers & - & 6 & - & - \\
BLSTM neurons & - & 64 & - & - \\
BLSTM dropout & - & 0 & - & - \\
Encoder attention heads & - & - & 3 & - \\
Encoder layers & - & - & 3 & - \\
Encoder neurons per layer & - & - & 0.1 & 2 \\
Encoder dropout & - & - & 2 & 64 \\
TSB layers & 2 & 2 & 64 & 128 \\
TSB neurons & 64 & 64 & 64 & 0.0001 \\
Batch size & 128 & 64 & 0.0005 & 0.0005 \\
Learning rate & 0.0005 & 0.0005 & 0.0005 & - \\
Weigth decay & 0.00005 & 0.00005 & & - \\
\hline
\end{tabular}

Abbreviations: DB, dense blocks; TSB, task-specific blocks.

\subsection{Evaluation}

\subsubsection{In-house triage protocol and baseline models}

First, to assess if DeepEMC ${ }^{2}$ provides an improvement in EMCI classification respect the existing clinical rules, performance metrics were obtained for the current in-house triage protocol of the Valencian emergency dispatch service.

Second, to compare the performance of the DL model respect well-known machine learning models in EMCI classification, we trained and evaluated the following baseline models:

- Multinomial naive bayes (NB) [84]: including a term of additive Laplace smoothing [85].

- Logistic regression (LR) [86]: including a penalty term for L2 regularization [87] and resorting to LBFGS [88] as optimizer algorithm.

- $\quad$ Random forest (RF) [89]: considering Gini impurity as splitting criterion [90], while assembling a total of 300 tree estimators whose maximum depth was equal to 50, being these optimal values determined via hyperparameter tuning procedures.

- Gradient boosting (GB) [91]: considering mean squared error with improvement score by Friedman [91] as splitting criterion, with a total of 300 tree estimators whose maximum depth was set in 5, being these optimal values determined by hyperparameter tuning processes.

Notably, the input data for these baseline models had to be adapted to be processed by them. Clinical variables were one-hot encoded instead of being fed as sequences of integers. Regarding free text observations, once spelling correction processes, subword tokenization and sentence truncation were carried out, subwords were one-hot encoded.

\subsubsection{Metrics}

Performance metrics were obtained in the test set (144 454 independent EMCI) for each label prediction task and each model trained-we recall here that EnsNet outputs are the same as DeepEMC ${ }^{2}$. The evaluation metrics included accuracy, recall, precision and F1-score [92,93]. For binary labels (life-threatening, jurisdiction), recall, precision and F1score were referencing the interest class-life-thread and emergency system jurisdiction. Regarding the multiclass label (response delay), recall and precision were calculated for each class and then averaged following a macro approach. Likewise, for all labels, macro F1-score [92,93] was computed, to dispose of a balanced multiclass performance descriptor-not influenced by class frequencies. Finally, for all metrics, $95 \%$ confidence intervals were calculated by 1000 bootstrap samples [94] extracted from the test set.

Metrics were calculated in the test set, for the protocol, the baseline models-naive bayes, logistic regression, random forest and gradient boosting - and the DL models developed-the ConNet, the CliNet, the TextNet and DeepEMC ${ }^{2}$. We recall here that, although DeepEMC ${ }^{2}$ is the definitive DL model which takes into account input data globally, results referring its constituting subnetworks, contrasted with baseline models trained with the same type of input data of each subnetwork, are also reported, to analyze the contribution of each set of inputs to the global model and where DL provides a substantial gaining over the other kind of models.

Likewise, percentage differences between DeepECM $^{2}$ and the protocol are also reported, as well as percentage differences between DeepECM ${ }^{2}$ and the best 
medRxiv preprint doi: https://doi.org/10.1101/2020.06.26.20123216; this version posted November 9, 2020. The copyright holder for this preprint (which was not certified by peer review) is the author/funder, who has granted medRxiv a license to display the preprint in perpetuity.

All rights reserved. No reuse allowed without permission.

baseline model - that baseline model with the best balanced multiclass performance-which has been measured in our work in terms of macro F1-score.

\section{Results}

Tables 3, 4 and 5 show the classification performance results for the life-threatening level, admissible response delay and emergency system jurisdiction labels, respectively.

\subsection{Life-threatening level}

Table 3 shows that DeepEMC ${ }^{2}$ - the global DL modelhighly outperforms the current protocol in the life-threatening prediction task with a $13.2 \%$ of accuracy improvement and a $12.5 \%$ of macro F1-score increment. This increment is statistically significant as reflected by the absence of overlapping in the $95 \%$ confidence intervals (CI). DeepEMC ${ }^{2}$ captures more true life-threatening situations-higher recallbeing much more precise-with less false positives.

In comparison to the baseline models, although DeepEMC $^{2}$ does not offer the best recall or precision, it achieves the best trade-off between them, as indicated by the best F1-score, being this metric statistically superior to those F1-scores attained by the baseline models. Likewise, referring to the best balanced two-class performance, DeepEMC ${ }^{2}$ presents the best macro F1-score, with statistically significant difference respect to the baselines models.

Focusing on the subnetworks, the ConNet is the weakest deep learning model. The CliNet offers the better detection rate for true life-threatening situations but at the expense of a significant amount of false positives. Finally, the TextNet exhibits the overall better behavior although its capability to capture true life-threatening events is not the best among the subnetworks.

Regarding the comparative performance among the subnetworks and their respective baseline models, it stands out the performance similitude among the ConNet and some of their associated baseline models as well as the high outcomes resemblance among the CliNet and the baseline models using clinical variables. Finally, notably the TextNet presents greater differences respect its corresponding baseline models, being these differences notorious in the F1-score and macro F1-score.

Table 3. Performances of the in-house triage protocol, baseline models and deep learning models in life-threatening prediction (test set). Bootstrapped $95 \%$ confidence intervals are shown between brackets. Percentage differences between DeepEMC ${ }^{2}$-the global deep learning model—and the protocol $\Delta \mathrm{P}(\%)$, along with percentage differences between DeepEMC ${ }^{2}$ and the best baseline model $\Delta \mathrm{BM}(\%)$-highest F1score and F1-score ${ }^{\mathrm{MACRO}}$ _ are also reported.

\begin{tabular}{|c|c|c|c|c|c|}
\hline \multirow{3}{*}{ Model } & \multicolumn{5}{|c|}{ Life-threatening level (yes/no) } \\
\hline & \multicolumn{3}{|c|}{ Single-class metrics (yes) } & \multicolumn{2}{|c|}{ Two-class metrics (yes/no) } \\
\hline & Recall & Precision & F1-score & Accuracy & F1-score ${ }^{\text {MACRO }}$ \\
\hline Protocol & $0.644[0.641,0.647]$ & $0.547[0.544,0.551]$ & $0.592[0.589,0.595]$ & $0.639[0.637,0.641]$ & $0.634[0.632,0.636]$ \\
\hline Context NB & $0.407[0.404,0.410]$ & $0.563[0.559,0.567]$ & $0.472[0.469,0.475]$ & $0.631[0.629,0.633]$ & $0.594[0.592,0.596]$ \\
\hline Context LR & $0.411[0.407,0.414]$ & $0.577[0.573,0.581]$ & $0.480[0.476,0.483]$ & $0.638[0.636,0.640]$ & $0.601[0.599,0.604]$ \\
\hline Context RF & $0.465[0.462,0.469]$ & $0.526[0.522,0.529]$ & $0.494[0.491,0.497]$ & $0.612[0.610,0.614]$ & $0.590[0.588,0.592]$ \\
\hline Context GB & $0.428[0.425,0.432]$ & $0.588[0.584,0.592]$ & $0.495[0.492,0.499]$ & $0.646[0.644,0.648]$ & $0.611[0.609,0.613]$ \\
\hline Context DL & $0.440[0.436,0.443]$ & $0.583[0.579,0.587]$ & $0.501[0.498,0.504]$ & $0.644[0.642,0.647]$ & $0.613[0.610,0.615]$ \\
\hline Clinical NB & $0.732[0.729,0.735]$ & $0.550[0.547,0.553]$ & $0.628[0.625,0.630]$ & $0.647[0.645,0.650]$ & $0.646[0.644,0.649]$ \\
\hline Clinical LR & $0.752[0.750,0.755]$ & $0.586[0.583,0.589]$ & $0.659[0.656,0.661]$ & $0.683[0.681,0.685]$ & $0.682[0.680,0.684]$ \\
\hline Clinical RF & $0.764[0.761,0.767]$ & $0.585[0.583,0.589]$ & $0.663[0.661,0.665]$ & $0.684[0.682,0.686]$ & $0.683[0.681,0.685]$ \\
\hline Clinical GB & $0.763[0.760,0.766]$ & $0.585[0.583,0.589]$ & $0.663[0.660,0.665]$ & $0.684[0.682,0.686]$ & $0.683[0.681,0.685]$ \\
\hline Clinical DL & $0.790[0.787,0.793]$ & $0.581[0.578,0.584]$ & $0.669[0.667,0.672]$ & $0.683[0.681,0.685]$ & $0.682[0.681,0.685]$ \\
\hline Text NB & $0.681[0.678,0.685]$ & $0.647[0.644,0.650]$ & $0.664[0.661,0.666]$ & $0.719[0.718,0.721]$ & $0.711[0.710,0.714]$ \\
\hline Text LR & $0.629[0.626,0.633]$ & $0.728[0.724,0.731]$ & $0.675[0.672,0.678]$ & $0.754[0.752,0.756]$ & $0.738[0.736,0.740]$ \\
\hline Text RF & $0.514[0.511,0.517]$ & $0.783[0.780,0.787]$ & $0.621[0.618,0.624]$ & $0.745[0.743,0.747]$ & $0.714[0.712,0.716]$ \\
\hline Text GB & $0.578[0.575,0.581]$ & $0.758[0.755,0.762]$ & $0.656[0.653,0.659]$ & $0.753[0.752,0.755]$ & $0.732[0.730,0.734]$ \\
\hline Text Net & $0.638[0.635,0.642]$ & $0.737[0.734,0.740]$ & $0.684[0.681,0.687]$ & $0.760[0.758,0.762]$ & $0.745[0.744,0.747]$ \\
\hline Global NB & $0.729[0.726,0.732]$ & $0.635[0.632,0.638]$ & $0.679[0.676,0.681]$ & $0.720[0.718,0.722]$ & $0.715[0.713,0.717]$ \\
\hline Global LR & $0.652[0.649,0.656]$ & $0.736[0.733,0.740]$ & $0.692[0.689,0.695]$ & $0.764[0.762,0.766]$ & $0.750[0.748,0.752]$ \\
\hline Global RF & $0.585[0.582,0.589]$ & $0.776[0.773,0.779]$ & $0.667[0.665,0.670]$ & $0.763[0.761,0.765]$ & $0.742[0.740,0.744]$ \\
\hline Global GB & $0.616[0.613,0.620]$ & $0.762[0.759,0.765]$ & $0.681[0.679,0.684]$ & $0.766[0.764,0.768]$ & $0.748[0.746,0.750]$ \\
\hline DeepEMC ${ }^{2}$ & $0.671[0.668,0.675]$ & $0.742[0.739,0.745]$ & $0.705[0.702,0.707]$ & $0.771[0.770,0.773]$ & $0.759[0.757,0.761]$ \\
\hline$\Delta \mathrm{P}(\%)$ & $2.7[2.1,3.4]$ & $19.5[18.8,20.1]$ & $11.3[10.7,11.8]$ & $13.2[12.9,13.6]$ & $12.5[12.1,12.9]$ \\
\hline$\Delta \mathrm{BM}(\%)$ & $1.9[1.2,2.6]$ & $0.6[-0.1,1.2]$ & $1.3[0.7,1.8]$ & $0.7[0.4,1.1]$ & $0.9[0.5,1.3]$ \\
\hline
\end{tabular}

Abbreviations: NB, naive bayes; LR, logistic regression; RF, random forest; GB, gradient boosting; DL, deep learning; $\Delta \mathrm{P}$, DeepEMC ${ }^{2}$ difference respect to the protocol; $\triangle \mathrm{BM}$, DeepEMC ${ }^{2}$ difference respect to the best baseline model in life-threatening prediction (logistic regression). 
medRxiv preprint doi: https://doi.org/10.1101/2020.06.26.20123216; this version posted November 9, 2020. The copyright holder for this preprint (which was not certified by peer review) is the author/funder, who has granted medRxiv a license to display the preprint in perpetuity.

All rights reserved. No reuse allowed without permission.

\subsection{Admissible response delay}

Table 4 shows that DeepEMC ${ }^{2}$ outcomes are significantly superior to those achieved by the protocol in the response delay prediction task (CI 95\%).

Overall detection of situations with a specific admissible response delay (undelayable, minutes, hours, days) is largely improved by DeepEMC ${ }^{2}-15.8 \%$ increment in macro recall-while remarkably enhancing overall precision $-17.3 \%$ increment. Regarding the general performance in all classes, DeepEMC ${ }^{2}$ significantly improves the protocol, with a $16.4 \%$ of accuracy improvement and a $17.5 \%$ of macro F1score increment.

DeepEMC ${ }^{2}$ does not offer the best overall precision compared to the baseline models. However, it improves the overall recall and the best balanced multiclass performance, in terms of macro F1-score. Furthermore, this global performance is the best, in terms of statistically significance difference respect the baseline models, although the performance difference respect the global gradient boosting model-best baseline model in admissible response delay prediction-is at the limit, since 0 is the lower bound of the 95\% confidence intervals for performance differences.

Focusing on DeepEMC ${ }^{2}$ subnetworks for response delay prediction, the ConNet is at the bottom in performance terms, not being capable of outperforming the protocol. The CliNet is clearly over the ConNet and already beats the protocol, while the TextNet is the best DeepEMC ${ }^{2}$ subnetwork in all metrics, with a substantial increase respect to the CliNet.

Regarding the comparative performance among the subnetworks and their respective baseline models, it can be appreciated the performance similitude among the ConNet and some of their associated baseline models as well as the high outcomes resemblance among the CliNet and the baseline models fed with the clinical variables. Finally, the TextNet presents greater differences respect its corresponding baseline models, being these differences significant in the macro F1score metric.

\subsection{Emergency system jurisdiction}

Table 5 shows that DeepEMC ${ }^{2}$ significantly outperforms the protocol in the jurisdiction prediction task $(95 \% \mathrm{CI})$. It captures more situations which are jurisdiction of the emergency system-better recall-being more precise-with less false positives. Respect to the overall performance in both classes, DeepEMC ${ }^{2}$ surpasses the protocol, with a $4.5 \%$ of accuracy improvement and a $5.1 \%$ of macro F1-score increment.

Table 4. Performances of the in-house triage protocol, baseline models and deep learning models in response delay prediction (test set). Bootstrapped 95\% confidence intervals are shown between brackets. Percentage differences between DeepEMC ${ }^{2}$ the global deep learning model—and the protocol $\Delta \mathrm{P}(\%)$, along with percentage differences between DeepEMC ${ }^{2}$ and the best baseline model $\Delta \mathrm{BM}(\%)$-highest F1score ${ }^{\mathrm{MACRO}}$ _ are also reported.

\begin{tabular}{|c|c|c|c|c|}
\hline \multirow{2}{*}{ Model } & \multicolumn{4}{|c|}{ Admissible response delay (undelayable, minutes, hours, days) } \\
\hline & Recall ${ }^{\text {MACRO }}$ & Precision ${ }^{\mathrm{MACRO}}$ & F1-score ${ }^{\text {MACRO }}$ & Accuracy \\
\hline Protocol & $0.411[0.409,0.413]$ & $0.416[0.414,0.419]$ & $0.401[0.398,0.403]$ & $0.428[0.426,0.430]$ \\
\hline Context NB & $0.375[0.373,0.377]$ & $0.382[0.379,0.385]$ & $0.364[0.362,0.366]$ & $0.396[0.394,0.399]$ \\
\hline Context LR & $0.376[0.374,0.378]$ & $0.396[0.393,0.398]$ & $0.369[0.367,0.371]$ & $0.406[0.403,0.408]$ \\
\hline Context RF & $0.348[0.345,0.350]$ & $0.357[0.354,0.359]$ & $0.350[0.348,0.352]$ & $0.371[0.369,0.373]$ \\
\hline Context GB & $0.382[0.380,0.384]$ & $0.414[0.411,0.417]$ & $0.383[0.381,0.385]$ & $0.415[0.413,0.417]$ \\
\hline Context DL & $0.376[0.374,0.378]$ & $0.415[0.412,0.418]$ & $0.377[0.374,0.379]$ & $0.413[0.411,0.415]$ \\
\hline Clinical NB & $0.458[0.456,0.460]$ & $0.503[0.501,0.506]$ & $0.460[0.458,0.462]$ & $0.482[0.480,0.484]$ \\
\hline Clinical LR & $0.479[0.477,0.481]$ & $0.522[0.520,0.525]$ & $0.488[0.486,0.490]$ & $0.505[0.503,0.507]$ \\
\hline Clinical RF & $0.477[0.475,0.479]$ & $0.533[0.530,0.535]$ & $0.485[0.483,0.488]$ & $0.507[0.504,0.509]$ \\
\hline Clinical GB & $0.477[0.475,0.479]$ & $0.532[0.530,0.535]$ & $0.485[0.483,0.488]$ & $0.507[0.504,0.509]$ \\
\hline Clinical DL & $0.477[0.475,0.479]$ & $0.530[0.527,0.532]$ & $0.485[0.483,0.487]$ & $0.506[0.504,0.508]$ \\
\hline Text NB & $0.527[0.524,0.529]$ & $0.517[0.515,0.519]$ & $0.519[0.517,0.521]$ & $0.533[0.531,0.535]$ \\
\hline Text LR & $0.544[0.542,0.546]$ & $0.564[0.562,0.567]$ & $0.550[0.548,0.553]$ & $0.569[0.567,0.572]$ \\
\hline Text RF & $0.524[0.522,0.527]$ & $0.583[0.581,0.586]$ & $0.535[0.533,0.538]$ & $0.563[0.561,0.566]$ \\
\hline Text GB & $0.545[0.543,0.547]$ & $0.577[0.575,0.580]$ & $0.554[0.552,0.556]$ & $0.574[0.572,0.576]$ \\
\hline Text DL & $0.544[0.542,0.546]$ & $0.583[0.580,0.585]$ & $0.555[0.553,0.557]$ & $0.576[0.574,0.578]$ \\
\hline Global NB & $0.537[0.534,0.539]$ & $0.531[0.529,0.534]$ & $0.533[0.531,0.535]$ & $0.549[0.547,0.551]$ \\
\hline Global LR & $0.557[0.555,0.559]$ & $0.579[0.577,0.581]$ & $0.564[0.562,0.567]$ & $0.582[0.580,0.585]$ \\
\hline Global RF & $0.547[0.545,0.549]$ & $0.593[0.590,0.595]$ & $0.557[0.555,0.560]$ & $0.581[0.579,0.583]$ \\
\hline Global GB & $0.562[0.560,0.565]$ & $0.593[0.591,0.596]$ & $0.572[0.570,0.574]$ & $0.589[0.587,0.592]$ \\
\hline DeepEMC $^{2}$ & $0.569[0.567,0.571]$ & $0.589[0.587,0.591]$ & $0.576[0.574,0.579]$ & $0.592[0.590,0.594]$ \\
\hline$\Delta \mathrm{P}(\%)$ & $15.8[15.4,16.2]$ & $17.3[16.8,17.7]$ & $17.5[17.1,18.1]$ & $16.4[16,16.8]$ \\
\hline$\Delta \mathrm{BM}(\%)$ & $0.7[0.2,1.1]$ & $-0.4[-0.9,0]$ & $0.4[0,0.9]$ & $0.3[-0.2,0.7]$ \\
\hline
\end{tabular}

Abbreviations: NB, naive bayes; LR, logistic regression; RF, random forest; GB, gradient boosting; DL, deep learning; $\Delta \mathrm{P}$, DeepEMC ${ }^{2}$ difference respect to the protocol; $\triangle \mathrm{BM}$, DeepEMC ${ }^{2}$ difference respect to the best baseline model in response delay prediction (gradient boosting). 
medRxiv preprint doi: https://doi.org/10.1101/2020.06.26.20123216; this version posted November 9, 2020. The copyright holder for this preprint (which was not certified by peer review) is the author/funder, who has granted medRxiv a license to display the preprint in perpetuity.

All rights reserved. No reuse allowed without permission.

DeepEMC ${ }^{2}$ does not offer the best recall or precision compared to the baseline models. However, it achieves, along with the gradient boosting model, the best trade-off between them, as indicated by their best F1-score, being this metric statistically superior to that attained by the logistic regression model — best baseline model in emergency system jurisdiction prediction. Likewise, referring to the best balanced two-class performance, DeepEMC ${ }^{2}$ presents the best macro F1-score, with statistically significant differences respect the baselines models.

Focusing on DeepEMC ${ }^{2}$ subnetworks, although the ConNet presents the highest recall values, its precision is not the best, with worse general results than the protocol in the jurisdiction prediction task. The CliNet provides a substantial

improvement over the later subnetwork, with an overall performance above the protocol. As in life-threatening and response delay, the TextNet is the subnetwork attaining the best outcomes.

Regarding to the comparative performance among the subnetworks and their respective baseline models, notably the performance is similar among the ConNet and some of their associated baseline models as well as the high outcomes resemblance among the CliNet and the baseline models fed with the clinical variables. Finally, it has to be highlighted that the TextNet presents greater differences respect its corresponding baseline models, being these differences notorious in the F1-score and accuracy.

Table 5. Performances of the in-house triage protocol, baseline models and deep learning models in jurisdiction prediction (test set). Bootstrapped 95\% confidence intervals are shown between brackets. Percentage differences between DeepEMC ${ }^{2}$ the global deep learning model — and the protocol $\Delta \mathrm{P}(\%)$, along with percentage differences between DeepEMC ${ }^{2}$ and the best baseline model $\Delta \mathrm{BM}(\%)$-highest F1score ${ }^{\mathrm{MACRO}}$ _ are also reported.

\begin{tabular}{|c|c|c|c|c|c|}
\hline \multirow{3}{*}{ Model } & \multicolumn{5}{|c|}{ Emergency system jurisdiction (yes/no) } \\
\hline & \multicolumn{3}{|c|}{ Single-class metrics (yes) } & \multicolumn{2}{|c|}{ Two-class metrics (yes/no) } \\
\hline & Recall & Precision & F1-score & Accuracy & F1-score ${ }^{\text {MACRO }}$ \\
\hline Protocol & $0.855[0.854,0.857]$ & $0.800[0.798,0.802]$ & $0.827[0.825,0.828]$ & $0.756[0.754,0.757]$ & $0.706[0.703,0.708]$ \\
\hline Context NB & $0.892[0.891,0.894]$ & $0.752[0.750,0.754]$ & $0.816[0.815,0.818]$ & $0.726[0.724,0.728]$ & $0.638[0.636,0.640]$ \\
\hline Context LR & $0.919[0.918,0.921]$ & $0.746[0.744,0.748]$ & $0.824[0.822,0.825]$ & $0.731[0.729,0.733]$ & $0.629[0.627,0.631]$ \\
\hline Context RF & $0.850[0.848,0.852]$ & $0.745[0.743,0.747]$ & $0.794[0.793,0.796]$ & $0.699[0.697,0.701]$ & $0.618[0.615,0.620]$ \\
\hline Context GB & $0.936[0.935,0.937]$ & $0.744[0.742,0.746]$ & $0.829[0.828,0.831]$ & $0.737[0.735,0.739]$ & $0.628[0.625,0.630]$ \\
\hline Context DL & $0.945[0.943,0.946]$ & $0.741[0.739,0.743]$ & $0.830[0.829,0.832]$ & $0.736[0.734,0.738]$ & $0.620[0.618,0.622]$ \\
\hline Clinical NB & $0.897[0.896,0.899]$ & $0.800[0.798,0.802]$ & $0.846[0.844,0.847]$ & $0.777[0.775,0.778]$ & $0.720[0.718,0.723]$ \\
\hline Clinical LR & $0.906[0.904,0.908]$ & $0.798[0.796,0.800]$ & $0.848[0.847,0.850]$ & $0.779[0.777,0.781]$ & $0.721[0.718,0.723]$ \\
\hline Clinical RF & $0.901[0.899,0.902]$ & $0.801[0.799,0.803]$ & $0.848[0.847,0.850]$ & $0.780[0.778,0.782]$ & $0.724[0.722,0.726]$ \\
\hline Clinical GB & $0.916[0.914,0.917]$ & $0.793[0.791,0.795]$ & $0.850[0.849,0.851]$ & $0.779[0.778,0.781]$ & $0.717[0.714,0.719]$ \\
\hline Clinical DL & $0.900[0.899,0.902]$ & $0.802[0.800,0.804]$ & $0.848[0.847,0.849]$ & $0.780[0.778,0.782]$ & $0.724[0.722,0.726]$ \\
\hline Text NB & $0.793[0.791,0.795]$ & $0.833[0.831,0.835]$ & $0.812[0.811,0.814]$ & $0.750[0.748,0.752]$ & $0.719[0.717,0.721]$ \\
\hline Text LR & $0.896[0.895,0.898]$ & $0.810[0.807,0.811]$ & $0.851[0.849,0.852]$ & $0.785[0.783,0.787]$ & $0.734[0.732,0.736]$ \\
\hline Text RF & $0.936[0.934,0.937]$ & $0.782[0.780,0.784]$ & $0.852[0.851,0.853]$ & $0.778[0.776,0.780]$ & $0.704[0.702,0.707]$ \\
\hline Text GB & $0.906[0.905,0.907]$ & $0.803[0.801,0.805]$ & $0.851[0.850,0.853]$ & $0.784[0.782,0.786]$ & $0.728[0.726,0.730]$ \\
\hline Text Net & $0.917[0.916,0.919]$ & $0.804[0.802,0.806]$ & $0.857[0.856,0.858]$ & $0.791[0.789,0.793]$ & $0.734[0.732,0.736]$ \\
\hline Global NB & $0.818[0.817,0.820]$ & $0.834[0.832,0.836]$ & $0.826[0.825,0.828]$ & $0.765[0.763,0.766]$ & $0.731[0.729,0.733]$ \\
\hline Global LR & $0.902[0.901,0.904]$ & $0.816[0.814,0.818]$ & $0.857[0.855,0.858]$ & $0.794[0.792,0.796]$ & $0.745[0.743,0.747]$ \\
\hline Global RF & $0.925[0.924,0.926]$ & $0.802[0.800,0.804]$ & $0.859[0.858,0.860]$ & $0.793[0.791,0.795]$ & $0.734[0.732,0.737]$ \\
\hline Global GB & $0.914[0.913,0.916]$ & $0.811[0.809,0.813]$ & $0.860[0.858,0.861]$ & $0.796[0.794,0.798]$ & $0.743[0.741,0.745]$ \\
\hline DeepEMC $^{2}$ & $0.895[0.894,0.897]$ & $0.827[0.825,0.829]$ & $0.860[0.858,0.861]$ & $0.801[0.799,0.802]$ & $0.757[0.755,0.759]$ \\
\hline$\Delta \mathrm{P}(\%)$ & $4[3.7,4.3]$ & $2.7[2.3,3.1]$ & $3.3[3,3.6]$ & $4.5[4.2,4.8]$ & $5.1[4.7,5.6]$ \\
\hline$\Delta \mathrm{BM}(\%)$ & $-0.7[-1,-0.4]$ & $1.1[0.7,1.5]$ & $0.3[0,0.6]$ & $0.7[0.3,1]$ & $1.2[0.8,1.6]$ \\
\hline
\end{tabular}

Abbreviations: NB, naive bayes; LR, logistic regression; RF, random forest; GB, gradient boosting; DL, deep learning; $\Delta \mathrm{P}$, DeepEMC ${ }^{2}$ difference respect to the protocol; $\triangle \mathrm{BM}$, DeepEMC ${ }^{2}$ difference respect to the best baseline model in jurisdiction prediction (logistic regression).

\section{Discussion}

\subsection{Relevance}

The superior performance of DeepEMC ${ }^{2}$ and some of the baseline models, respect to the in-house triage protocol, suggests the existence of information provided during the emergency medical call not considered by the current protocol, but captured by the machine learning models. Likewise, the DL approach is preferable over the other families of models tested, since DeepEMC ${ }^{2}$ outcomes are significantly above those attained by the baseline models.

In referring context and clinical variables, DL is not clearly at the top. However, regarding the free text dispatcher observations, the DL approach is, overall, remarkably superior. Likewise, as TextNet outcomes are far better than those attained by the ConNet and CliNet, the most valuable information provided during the emergency medical call 
medRxiv preprint doi: https://doi.org/10.1101/2020.06.26.20123216; this version posted November 9, 2020. The copyright holder for this

preprint (which was not certified by peer review) is the author/funder, who has granted medRxiv a license to display the preprint in perpetuity.

All rights reserved. No reuse allowed without permission.

would be present at these unstructured features. Since text fields are unbounded, they would embrace wider casuistry, allowing more precision in the EMCI description, lowering, consequently, its uncertainty.

Regarding the clinical variables, they stand as an excellent life-threatening detector features-about $80 \%$ of total cases. This could be due to the fact that dispatchers ask for them to reduce chances of missing situations where patient's life is at risk. Similarly, the outstanding emergency system jurisdiction recall of demographics and circumstantial factors-capturing about $95 \%$ of total cases - may be related with patient profiles highly susceptible from requiring emergency aid, e.g., elderly cardiac patient males.

Comparing classification scores across tasks, the hardest classification problem appeared to predict the admissible response delay, probably derived from the fact that it is a multiclass label, presenting twice possible outputs (undelayable, minutes, hours, days) than the other labels (lifethreatening, jurisdiction), which are binary.

The modular approach followed in this work, assembling four specialized subnetworks into a single global network (DeepEMC ${ }^{2}$ ), has shown that the potential of the aggregated network is superior to any of its individual components, balancing their respective weaknesses and strengths while properly integrating processed information within each one.

Finally, the results of this work imply that current emergency dispatch processes could be improved by means of deep learning, eventually deriving in a positive impact over patient wellbeing and health services sustainability.

\subsection{Limitations}

The main limitation of this work is the inherent uncertainty bound to the problem: in the studied dataset it was likely to find similar input combinations presenting completely different label values. In other words, the challenge faced in this work exhibits classes overlap, where different disorders may present the same clinical picture. For example, chest pain may imply a life-threatening situation, if the underlying unknown cause is a heart attack, or not, since it could be derived from a prior anxiety crisis. This nondiscriminative variability sets bounds in terms of maximum performance attainable by any model-Bayes error [95].

Besides, the data available to conduct this work lies between 2009 and 2012 years (both included). Even though the clinical framework of pathologies like heart failure or epileptic crisis could be fairly constant across time, an in-depth study of potential dataset shifts [96,97], and related abrupt or gradual changes regarding the statistical distributions of new data [98] has to be carried out before implementing the model in emergency medical dispatch centers.

\subsection{Future work}

Next steps include the evaluation of DeepEMC ${ }^{2}$ with prospective cases from the Valencia region-with more recent incidents, monitoring the aforementioned dataset shifts and acting in consequence. Passing this phase favorably would enable us to begin the integration of the model in an emergency medical dispatch center, with a prospective evaluation of the system performance and added value on routine settings through a randomized controlled trial for CDSS [99,100]. To accomplish that, a graphical user interface will be proposed, to allow the interaction between the dispatcher and the model during the call. Finally, the resulting tool will be implemented in the emergency medical dispatch center of the Valencian Community.

\section{Conclusions}

A novel deep ensemble multitask model (DeepEMC ${ }^{2}$ ) designed to aid non-clinical dispatchers during emergency medical calls to classify incidents by their lifethreatening level, admissible response delay and emergency system jurisdiction, has been developed and successfully evaluated. To our knowledge, this is the first deep learning model implemented to face this challenge.

The performance achieved by the model is highly superior to that attained by the current in-house triage protocol of the emergency medical dispatch service of the Valencian Community, achieving a macro F1-score improvement of $12.5 \%, 17.5 \%, 5.1 \%$ in life-threatening, response delay and jurisdiction classification, respectively. Likewise, DeepEMC ${ }^{2}$ outcomes are above those accomplished by the additional machine learning models tested, including naive bayes, logistic regression, random forest and gradient boosting. This increment was proved as statistically significant $(\alpha=0.05)$.

Remarkably, the network modular design with specialized subnetworks for the different data modalities has allowed discovering the potential benefit of the information contained in free text fields for the automatic classification of emergency medical call incidents. This information can be used to optimize current guidelines.

The implantation of this model in medical dispatch centers would have a remarkable impact in patient wellbeing and health services sustainability.

\section{Funding}

This work has been supported by the Valencian agency for security and emergency response project A180017304-1, the Ministry of Science, Innovation and Universities of Spain program FPU18/06441 and the EU Horizon 2020 project InAdvance 825750.

\section{Declaration of competing interest}

The authors declare no competing interests. 
medRxiv preprint doi: https://doi.org/10.1101/2020.06.26.20123216; this version posted November 9, 2020. The copyright holder for this preprint (which was not certified by peer review) is the author/funder, who has granted medRxiv a license to display the preprint in perpetuity.

\section{All rights reserved. No reuse allowed without permission.}

\section{Acknowledgements}

We thank the support of physicians from the Health Services Department of Valencian Community. CS acknowledges the support of NVIDIA GPU Grant Program.

\section{References}

[1] J. J. Clawson and K. B. Dernocoeur, "Principles of emergency medical dispatch," Priority Press, 2003.

[2] A. Blandford and B. W. Wong, "Situation awareness in emergency medical dispatch," International journal of human-computer studies 61.4, pp. 421-452, 2004.

[3] S. J. Stratton, "Triage by emergency medical dispatchers," Prehospital and disaster medicine, pp. 263-269, 1992.

[4] J. J. Clawson, "Dispatch priority training: strengthening the weak link," JEMS, pp. 32-35 (6), 1981.

[5] L. Palumbo, J. Kubincanek, C. Emerman, N. Jouriles, R. Cydulka and B. Shade, "Performance of a system to determine EMS dispatch priorities," The American journal of emergency medicine, pp. 388-390 (14), 1996.

[6] L. Weibel, I. Gabrion, M. Aussedat and G. Kreutz, "Work-related stress in an emergency medical dispatch center," Annals of emergency medicine, pp. 500-506 (41), 2003.

[7] K. Forslund, A. Kihlgren and M. Kihlgren, "Operators' experiences of emergency calls.," Journal of telemedicine and telecare, pp. 290-297 (10), 2004.

[8] B. Ek, P. Edström, A. Toutin and M. Svedlund, "Reliability of a Swedish pre-hospital dispatch," International emergency nursing, pp. 143-149, 2013.

[9] J. Leprohon and V. L. Patel, "Decision-making strategies for telephone triage in emergency medical services," Medical Decision Making, pp. 240-253 (15), 1995.

[10] M. Srámek, W. Post and R. W. Koster, "Telephone triage of cardiac emergency calls by dispatchers: a prospective study of 1386 emergency calls," Heart, pp. 440-445 (71), 1994.

[11] Institute of Medicine Committee on the Future of Emergency Care in the US Health System, "Emergency medical services: at the crossroads," Washington: DC, 2006.

[12] L. Hjälte, B.-O. Suserud, J. Herlitz and I. Karlberg, "Why are people without medical needs transported by ambulance? A study of indications for pre-hospital care," European Journal of Emergency Medicine, pp. 151-156 (14), 2007.

[13] Agency for healthcare research and quality, "Emergency severity index implementation handbook," 2012.

[14] Manchester triage group, "Emergency triage," 2014.

[15] Échelle de triage et de gravité, "Revisions to the Canadian emergency department triage and acuity scale (CTAS). Guidelines 2016," 2016.
[16] Australasian College for Emergency Medicine, "A National Triage Scale for Australian Emergency Departments [position paper]," Melbourne, Victoria, Australia: Australasian College for Emergency Medicine, 1993.

[17] M. Christ, F. Grossmann, D. Winter, R. Bingisser and E. Platz, "Modern triage in the emergency department," Deutsches Ärzteblatt International, p. 892 (107), 2010.

[18] M. N. Storm-Versloot, D. T. Ubbink, J. Kappelhof and J. S. Luitse, "Comparison of an informally structured triage system, the emergency severity index, and the manchester triage system to distinguish patient priority in the emergency department," Academic Emergency Medicine, pp. 822-829 (18), 2011.

[19] N. Seiger, M. van Veen, E. W. Steyerberg, M. Ruige, A. H. Van Meurs and H. A. Moll, "Undertriage in the Manchester triage system: an assessment of severity and options for improvement," Archives of disease in childhood, pp. 653-657 (96), 2011.

[20] J. M. Zachariasse, N. Seiger, P. P. Rood, C. F. Alves, P. Freitas, F. J. Smit, G. R. Roukema and H. A. Moll, "Validity of the Manchester Triage System in emergency care: A prospective observational study," PloS one, 2017.

[21] G. FitzGerald, G. A. Jelinek, D. Scott and M. F. Gerdtz, "Emergency department triage revisited," Emergency Medicine Journal, pp. 86-92 (27), 2010.

[22] L. Farand, J. Leprohon, M. Kalina, F. Champagne, A. P. Contandriopoulos and A. Preker, "The role of protocols and professional judgement in emergency medical dispatching," European journal of emergency medicine: official journal of the European Society for Emergency Medicine, pp. 136-148 (2), 1995

[23] Y. LeCun, Y. Bengio and G. Hinton, "Deep learning," Nature, pp. 436-444 (521) , 2015.

[24] G. Hinton, L. Deng, D. Yu, G. E. Dahl, A.-R. Mohamed, N. Jaitly, A. Senior, V. Vanhoucke, P. Nguyen, T. N. Sainath and B. Kingsbury, "Deep neural networks for acoustic modeling in speech recognition: The shared views of four research groups," IEEE Signal processing magazine, pp. 82-97 (29), 2012.

[25] O. Russakovsky, J. Deng, H. Su, J. Krause, S. Satheesh, S. Ma, Z. Huang, A. Karpathy, A. Khosla, M. Bernstein, A. C. Berg and L. Fei-Fei, "Imagenet large scale visual recognition challenge," International journal of computer vision, pp. 211-252, 2015.

[26] J. Hirschberg and C. D. Manning, "Advances in natural language processing," Science, pp. 261-266 (349), 2015.

[27] D. Silver, A. Huang, C. J. Maddison, A. Guez, L. Sifre, G. van den Driessche, J. Schrittwieser, I. Antonoglou, V. Panneershelvam, M. Lanctot, S. Dieleman, D. Grewe, J. Nham, N. Kalchbrenner, I. Sutskever, T. Lillicrap, M. Leach, K. Kavukcuoglu, T. Graepel and D. Hassabis, "Mastering the game of Go with deep neural networks and tree search," nature, p. 484, 2016. 
medRxiv preprint doi: https://doi.org/10.1101/2020.06.26.20123216; this version posted November 9, 2020. The copyright holder for this preprint (which was not certified by peer review) is the author/funder, who has granted medRxiv a license to display the preprint in perpetuity.

All rights reserved. No reuse allowed without permission.

[28] M. S. Maxwell, S. G. Henderson and H. Topaloglu, "Ambulance redeployment: An approximate dynamic programming approach," Winter Simulation Conference, pp. 1850-1860, 2009

[29] L. A. McLay and M. E. Mayorga, "A model for optimally dispatching ambulances to emergency calls with classification errors in patient priorities," IIE Transactions, pp. 1-24 (45), 2013.

[30] A. Y. Chen and T.-Y. Lu, "A GIS-based demand forecast using machine learning for emergency medical services," Computing in Civil and Building Engineering, pp. 1634-1641, 2014.

[31] N. Channouf, P. L'Ecuyer, A. Ingolfsson and A. N. Avramidis, "The application of forecasting techniques to modeling emergency medical system calls in Calgary, Alberta," Health care management science, pp. 25-45 (10), 2007.

[32] I. Lefter, L. J. Rothkrantz, D. A. van Leeuwen and P. Wiggers, "Automatic stress detection in emergency(telephone) calls," International Journal of Intelligent Defence Support Systems, pp. 148-168 (4), 2011.

[33] F. Barrientos and G. Sainz, "Interpretable knowledge extraction from emergency call data based on fuzzy unsupervised decision tree," Knowledge-based systems, pp. 77-87 (25), 2012.

[34] P. Klement and V. Snásel, "Using SOM in the performance monitoring of the emergency," Simulation Modelling Practice and Theory, pp. 98-109 (19), 2011.

[35] S. N. Blomberg, F. Folke, A. K. Ersboll, H. C. Christensen, C. Torp-Pedersen, M. R. Sayre, C. R. Counts and K. L. Freddy, "Machine learning as a supportive tool to recognize cardiac arrest in emergency calls," Resuscitation, pp. 322-329, 2019.

[36] L. Tollinton, A. M. Metcalf and S. Velupillai, "Enhancing predictions of patient conveyance using emergency call handler free text notes for unconscious and fainting incidents reported to the London Ambulance Service," International Journal of Medical Informatics, no. 104179, 2020 .

[37] World health organization, "International classification of diseases, ICD-9," 2015.

[38] N. C. Dalkey, "The Delphi method: an experimental study of group opinion," RAND CORP SANTA MONICA CALIF, 1969.

[39] C. Sáez, S.-T. Liaw, E. Kimura, P. Coorevits and J. M. García-Gómez, "Guest editorial: Special issue in biomedical data quality assessment methods," Computer methods and programs in biomedicine, vol. 181, 2019.

[40] C. Sáez, P. Pereira Rodrigues, J. Gama, M. Robles and J. M. García-Gómez, "Probabilistic change detection and visualization methods for the assessment of temporal stability in biomedical data quality," Data Mining and Knowledge Discovery, pp. 950-975 (29), 2015.
[41] C. Sáez, M. Robles and J. M. García-Gómez, "Stability metrics for multi-source biomedical data based on simplicial projections from probability distribution distances," Statistical methods in medical research, pp. 312-336 (26), 2017.

[42] C. Sáez, O. Zurriaga, J. Pérez-Penadés, I. Melchor, M. Robles and J. M. García-Gómez, "Applying probabilistic temporal and multisite data quality control methods to a public health mortality registry in Spain: a systematic approach to quality control of repositories," Journal of the American Medical Informatics Association, vol. 23, no. 6, pp. 10851095, 2016.

[43] Python Software Foundation, "Python Language Reference, version 3.6.5," python.org, 2018.

[44] W. McKinney, "Data structures for statistical computing in python," Proceedings of the 9th Python in Science Conference, pp. 51-56 (445), 2010.

[45] S. v. d. Walt, S. C. Colbert and G. Varoquaux, "The NumPy Array: A Structure for Efficient Numerical Computation," Computing in Science \& Engineering, pp. 2230 (13), 2011.

[46] J. Gonzalez, P. Rodrigues and A. Cohen, "Fuzzywuzzy: Fuzzy string matching in python," 2017.

[47] F. Pedregosa, G. Varoquaux, A. Gramfort, V. Michel, B. Thirion, O. Grisel, M. Blondel, P. Prettenhofer, R. Weiss, V. Dubourg, J. Vanderplas, A. Passos, D. Cournapeau, M. Brucher, M. Perrot and É. Duchesnay, "Scikit-learn: Machine learning in Python," the Journal of machine Learning research, vol. 12, pp. 2825-2830, 2011.

[48] A. Paszke, S. Gross, S. Chintala, G. Chanan, E. Yang, Z. De Vito, Z. Lin, A. Desmaison, L. Antiga and A. Lerer, "Automatic differentiation in pytorch," 31st Conference on Neural Information Processing Systems (NIPS), 2017.

[49] T. Wolf, L. Debut, V. Sanh, J. Chaumond, C. Delangue, A. Moi, P. Cistac, T. Rault, R. Louf, M. Funtowicz and J. Brew, "HuggingFace's Transformers: State-of-the-art Natural Language Processing," Huggingface's transformers: State-of-the-art natural language processing, pp. ArXiv, abs/1910.03771, 2019.

[50] J. Bergstra, B. Komer, C. Eliasmith, D. Yamins and D. D. Cox, "Hyperopt: a python library for model selection and hyperparameter optimization," ICML Workshop on Automated Machine Learning, p. (9), 2014.

[51] L. A. Zadeh, "Fuzzy sets," Information and control, pp. 338-353 (8), 1965.

[52] V. Novák, I. Perfilieva and J. Mockor, "Mathematical principles of fuzzy logic," Springer Science \& Business Media, p. Vol. 517, 2012.

[53] M. Dwarampudi and N. V. Reddy, "Effects of padding on LSTMs and CNNs," arXiv preprint arXiv:1903.07288, 2019. 
medRxiv preprint doi: https://doi.org/10.1101/2020.06.26.20123216; this version posted November 9, 2020. The copyright holder for this preprint (which was not certified by peer review) is the author/funder, who has granted medRxiv a license to display the preprint in perpetuity.

All rights reserved. No reuse allowed without permission.

[54] R. A. Wagner and M. J. Fischer, "The string-to-string correction problem," Journal of the ACM (JACM), pp. 168173 (21), 1974.

[55] Y. Wu, M. Schuster, Z. Chen, Q. V. Le and M. Norouzi, "Google's neural machine translation system: Bridging the gap between human and machine translation.," arXiv preprint arXiv:1609.08144, 2016.

[56] R. Kohavi, "A study of cross-validation and bootstrap for accuracy estimation and model selection," Ijcai, pp. 11371145 (14), 1995.

[57] K. Chen, "Deep and modular neural networks," Handbook of Computational Intelligence, pp. 473-494, 2015.

[58] R. Caruana, "Multitask learning," Machine learning, pp. 41-75 (28), 1997.

[59] S. Ruder, "An Overview of Multi-Task Learning in Deep Neural Networks," arXiv preprint arXiv:1706.05098, 2017.

[60] F. Rosenblatt, "Principles of neurodynamics. perceptrons and the theory of brain mechanisms," Cornell Aeronautical Lab Inc Buffalo NY, pp. No. VG-1196-G-8, 1961.

[61] I. Goodfellow, Y. Bengio and A. Courville, "Deep learning," MIT press, 2016.

[62] S. Ioffe and C. Szegedy, "Batch normalization: Accelerating deep network training by reducing internal covariate shift," arXiv preprint arXiv:1502.03167 , 2015.

[63] A. L. Maas, A. Y. Hannun and A. Y. Ng, "Rectifier nonlinearities improve neural network acoustic models," Proc. icml, 2013.

[64] C. Nwankpa, W. Ijomah, A. Gachagan and S. Marshall, "Activation functions: Comparison of trends in practice and research for deep learning," arXiv preprint arXiv:1811.03378, 2018.

[65] G. Hinton, N. Srivastava, A. Krizhevsky, I. Sutskever and R. Salakhutdinov, "Improving neural networks by preventing co-adaptation of feature detectors," arXiv preprint arXiv:1207.0580, 2012.

[66] Y. Bengio, R. Ducharme, V. Pascal and C. Jauvin, "A neural probabilistic language model," Journal of machine learning research, pp. 1137-1155 (3), 2003.

[67] M. Schuster and P. K. Kuldip, "Bidirectional recurrent neural networks," IEEE Transactions on Signal Processing, pp. 2673-2681 (45), 1997.

[68] K. He, X. Zhang, S. Ren and J. Sun, "Deep residual learning for image recognition," Proceedings of the IEEE conference on computer vision and pattern recognition, pp. 770-778, 2016.

[69] J. Devlin, M.-W. Chang, K. Lee and K. Toutanova, "Bert: Pre-training of deep bidirectional transformers for language understanding.," arXiv preprint arXiv:1810.04805, 2018.
[70] A. Vaswani, N. Shazeer, N. Parmar, J. Uszkoreit, L. Jones, A. N. Gomez, Ł. Kaiser and I. Polosukhin, "Attention is all your need," Advances in neural information processing systems, pp. 5998-6008, 2017.

[71] D. P. Kingma and J. L. Ba, "Adam: A method for stochastic optimization," International Conference on Learning Representations (ICLR), 2015.

[72] S. Ruder, "An overview of gradient descent optimization algorithms," arXiv preprint arXiv:1609.04747, 2016.

[73] S. Sun, Z. Cao, H. Zhu and J. Zhao, "A Survey of Optimization Methods from a Machine Learning Perspective," IEEE transactions on cybernetics, 2019.

[74] A. Krogh and J. A. Hertz, "A simple weigth decay can improve generalization," Advances in neural information processing systems, pp. 950-957, 1992.

[75] D. P. Bertsekas, "Incremental least squares methods and the extended Kalman filter," SIAM Journal on Optimization, pp. 807-822 (6), 1996.

[76] R. Hecht-Nielsen, "Theory of the backpropagation neural network," Neural networks for perception. Academic Press, pp. 65-93, 1992.

[77] P. J. Werbos, "Backpropagation through time: what it does and how to do it," Proceedings of the IEEE , pp. 1550$1560,1990$.

[78] K. Janocha and W. M. Czarnecki, "On Loss Functions for Deep Neural Networks in Classification," arXiv preprint arXiv:1702.05659, 2017.

[79] K. He, X. Zhang, S. Ren and J. Sun, "Delving deep into rectifiers: Surpassing human-level performance on imagenet classification," Proceedings of the IEEE international conference on computer vision, pp. 1026-1034, 2015.

[80] X. Glorot and Y. Bengio, "Understanding the difficulty of training deep feedforward neural networks," Proceedings of the thirteenth international conference on artificial intelligence and statistics, pp. 249-256, 2010.

[81] B. Settles, "Active learning literature survey," University of Wisconsin-Madison Department of Computer Sciences, 2009.

[82] J. Bergstra, R. Bardenet, Y. Bengio and B. Kégl, "Algorithms for hyper-parameter optimization," Advances in neural information processing systems, pp. 2546-2554, 2011.

[83] D. R. Jones, "A taxonomy of global optimization methods based on response surfaces," Journal of global optimization, pp. 345-383 (21), 2001.

[84] T. Bayes, "LII. An essay towards solving a problem in the doctrine of chances. By the late Rev. Mr. Bayes, FRS communicated by Mr. Price, in a letter to John Canton, AMFR S," Philosophical transactions of the Royal Society of London, vol. 53, pp. 370-418, 1763. 
medRxiv preprint doi: https://doi.org/10.1101/2020.06.26.20123216; this version posted November 9, 2020. The copyright holder for this preprint (which was not certified by peer review) is the author/funder, who has granted medRxiv a license to display the preprint in

All rights reserved. No reuse allowed without permission.

[85] C. D. Manning, H. Schütze and P. Raghavan, "Introduction to information retrieval," Cambridge university press, 2008.

[86] J. A. Nelder and R. W. Wedderburn, "Generalized linear models," Journal of the Royal Statistical Society: Series A (General), vol. 135, no. 3, pp. 370-384, 1972.

[87] A. Y. Ng, "Feature selection, L1 vs. L2 regularization, and rotational invariance," Proceedings of the twenty-first international conference on Machine learning. 2004, p. 78, 2004.

[88] D. C. Liu and J. Nocedal, "On the limited memory BFGS method for large scale optimization," Mathematical programming , vol. 45, pp. 503-528, 1989.

[89] T. K. Ho, "Random decision forests," Proceedings of 3rd international conference on document analysis and recognition, pp. 278-282 (1), 1995.

[90] L. E. Raileanu and K. Stoffel, "Theoretical comparison between the gini index and information gain criteria," Annals of Mathematics and Artificial Intelligence, vol. 41, no. 1, pp. 77-93, 2004.

[91] J. H. Friedman, "Greedy function approximation: a gradient boosting machine," Annals of statistics, pp. 11891232, 2001.

[92] Y. Yang and X. Liu, "A re-examination of text categorization methods," Proceedings of the 22nd annual international ACM SIGIR conference on Research and development in information retrieval, pp. 42-49, 1999.

[93] G. Tsoumakas, I. Katakis and I. Vlahavas, "Mining multi-label data," Data mining and knowledge discovery handbook. Springer, Boston, MA., pp. 667-685, 2009.
[94] B. Efron and R. J. Tibshirani, "An introduction to the bootstrap," CRC press, 1994.

[95] K. Fukunaga, "Introduction to statistical pattern recognition," Elsevier, 2013.

[96] J. Quionero-Candela, M. Sugiyama, A. Schwaighofer and N. D. Lawrence, "Dataset shift in machine learning," The MIT Press, 2009.

[97] C. Sáez, A. Gutiérrez-Sacristán, I. Kohane, J. M. García-Gómez and P. Avillach, "EHRtemporalVariability: delineating temporal dataset shifts in electronic health records," GigaScience, vol. 9, no. 8, 2020.

[98] C. Sáez and J. M. García-Gómez, "Kinematics of big biomedical data to characterize temporal variability and seasonality of data repositories: functional data analysis of data temporal evolution over non-parametric statistical manifolds," International journal of medical informatics, vol. 119, pp. 109-124, 2018.

[99] C. Sáez, L. Martí-Bonmatí, Á. Alberich-Bayarri, M. Robles and J. M. García-Gómez, "Randomized pilot study and qualitative evaluation of a clinical decision support system for brain tumour diagnosis based on SV 1H MRS: Evaluation as an additional information procedure for novice radiologists," Computers in biology and medicine, vol. 45, no. 26-33, 2014.

[100] D. C. Angus, "Randomized Clinical Trials of Artificial Intelligence," Jama, vol. 323, no. 11, pp. 1043-1045, 2020. 